\title{
LES CELLULES SOLAIRES ULTRAFINES
}

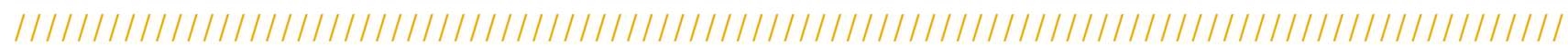

\section{Stéphane COLLIN ${ }^{1,2, *}$, Andrea CATTONI ${ }^{1,2}$}

${ }^{1}$ Centre de Nanosciences et de Nanotechnologies, CNRS, Université Paris-Saclay, Palaiseau, France

${ }^{2}$ Institut Photovoltaïque d'Ile-de-France, Palaiseau, France

*stephane.collin@c2n.upsaclay.fr

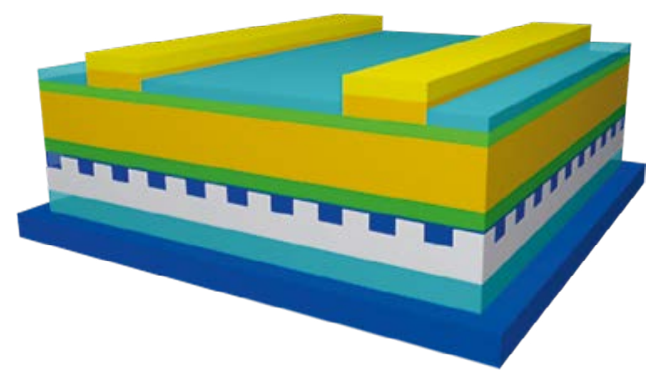

\begin{abstract}
Le photovoltaïque est devenu compétitif par rapport aux autres sources d'énergie. Il reste néanmoins beaucoup de progrès technologiques possibles. Aujourd'hui, la diminution de l'épaisseur des cellules solaires peut devenir l'un des éléments clés pour développer des cellules solaires à la fois moins chères et plus efficaces. Nous proposons une revue de l'état de l'art des cellules solaires ultrafines, des stratégies pour marier nanophotonique et photovoltaïque, et des perspectives offertes par la démonstration récente de cellules solaires de près de $20 \%$ d'efficacité et seulement de $200 \mathrm{~nm}$ d'épaisseur.
\end{abstract}

https://doi.org/10.1051/photon/202010244

Article publié en accès libre sous les conditions définies par la licence Creative Commons Attribution License CC-BY (http://creativecommons.org/licenses/by/4.0), qui autorise sans restrictions l'utilisation, la diffusion, et la reproduction sur quelque support que ce soit, sous réserve de citation correcte de la publication originale.

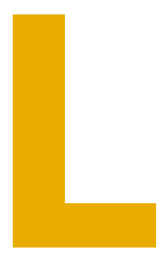

e photovoltaïque (PV) constitue certainement l'un des secteurs de l'optoélectronique qui connaît actuellement la plus forte croissance, avec une augmentation des capacités installées d'environ $30 \%$ par an depuis plus de 5 ans. La chute vertigineuse du prix des modules $\mathrm{PV}$ (moins de 0,25 €/Wc début 2020) est une bonne nouvelle pour accélérer la transition énergétique et espérer atteindre les objectifs de la COP21. Le coût actualisé de l'énergie (LCOE) photovoltaïque est maintenant égal voire inférieur à celui des centrales électriques conventionnelles. Il reste néanmoins beaucoup de progrès possibles pour améliorer la technologie $\mathrm{PV}$ et la rendre encore plus rentable et durable.
La réduction des prix nécessite une augmentation de l'efficacité des cellules solaires et une réduction de leurs coûts de fabrication [1,2]. $\mathrm{Au}$ cours des 10 dernières années, l'efficacité moyenne des modules commerciaux en silicium (environ $95 \%$ du marché) a progressé de plus de $5 \%$ pour atteindre environ $20 \%$ aujourd'hui, alors que les cellules de laboratoire atteignent $26,7 \%$, avec une limite théorique de $29,4 \%$ pour ce matériau. Les coûts de fabrication ont bénéficié d'économies d'échelle, en combinaison avec le développement de procédés plus efficaces. Aujourd'hui, la diminution de l'épaisseur des cellules solaires peut devenir l'un des éléments clés pour développer des cellules solaires à la fois moins chères et plus efficaces. A cause de la faible absorption des semiconducteur à bande interdite (Eg) indirecte, l'épaisseur typique des cellules solaires en silicium est de $150 \mu \mathrm{m}$, mais une réduction d'un facteur 10 à 50 est envisageable en utilisant un piégeage optique efficace grâce à une texturation aléatoire ou périodique. Elle pourrait également accroître la compétitivité des autres filières photovoltaïques dites de couches minces, formées de semiconducteurs polycristallins (CdTe ou CIGS, alliage de $\left.\mathrm{Cu}(\mathrm{In}, \mathrm{Ga})(\mathrm{S}, \mathrm{Se})_{2}\right)$ ou monocristallins III-V (GaAs par exemple) de 2 à $4 \mu \mathrm{m}$ d'épaisseur.

Nous proposons une revue de létat de l'art des cellules ultrafines, des stratégies de piégeage optique qui permettent de compenser la diminution de l'absorption avec l'épaisseur, et des défis à relever pour tirer le meilleur parti du potentiel de la 
nanophotonique. Nous présenterons ensuite les résultats récents qui ouvrent de nouvelles perspectives pour la réalisation de cellules solaires ultrafines de plus de $20 \%$ d'efficacité.

\section{ETAT DE L'ART DES CELLULES SOLAIRES ULTRAFINES}

La figure 1(a) montre la densité de courant de court-circuit Jsc en fonction de l'épaisseur de cellules solaires en silicium sous illumination solaire. Jsc est une figure de mérite qui représente l'efficacité d'un dispositif à absorber les photons du spectre solaire, puis à collecter les charges dans un circuit externe. Différentes stratégies de piégeage de la lumière peuvent être mises en œuvre pour améliorer l'absorption optique (Encart). Jsc diminue avec l'épaisseur de silicium, mais la diffusion lambertienne doit théoriquement permettre de maintenir des valeurs à l'état de l'art, autour de $40 \mathrm{~mA} / \mathrm{cm}^{2}$, avec une épaisseur de l'ordre de $10 \mu \mathrm{m}$, au lieu des $>100 \mu \mathrm{m}$ usuels. Cette figure montre de nombreux résultats expérimentaux de la littérature (triangles pleins) pour des épaisseurs de $1 \mu \mathrm{m}$ à $100 \mu \mathrm{m}$. On observe que la quasi-totalité de ces résultats restent proches de ce qui est attendu pour l'absorption simple passage, avec seulement quatre résultats qui dépassent le double passage. Pourtant, des résultats numériques montrent qu'il est possible de faire beaucoup mieux, et même de dépasser le modèle de diffusion lambertienne (triangles blancs).

Pourquoi observe-t-on une telle différence entre les performances expérimentales et les prédictions théoriques ? La figure 2(b) montre les principales sources de pertes optiques dans une cellule solaire. Le premier phénomène parasite est une réflexion sur la face avant de la cellule, soit sur les contacts métalliques (ombrage), soit à cause d'un revêtement anti-reflet imparfait. La lumière qui entre dans la cellule traverse ensuite la couche fenêtre, qui joue le rôle de contact sélectif pour un type de porteurs
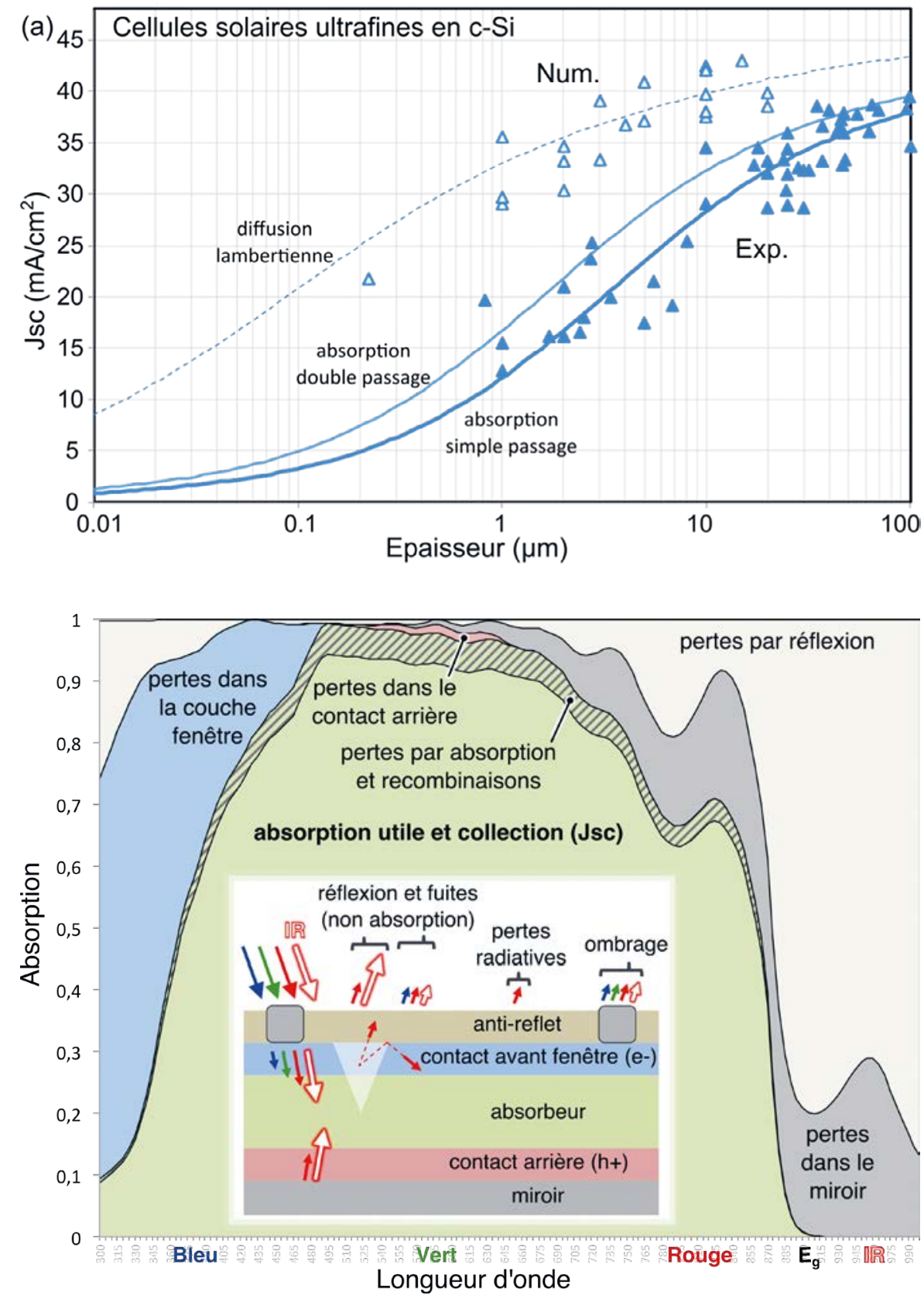

de charge (électrons). Cette couche est typiquement formée d'un semiconducteur à grand gap ou d'un oxyde transparent conducteur. Idéalement transparente, elle génère néanmoins de l'absorption parasite pour les photons les plus énergétiques (zone bleue).

Aux grandes longueurs d'onde, la lumière est réfléchie sur le miroir après avoir traversé la couche de contact sélectif rouge en face arrière. Si des phénomènes de
Figure 1.

Performances de cellules solaires ultrafines en silicium en fonction de l'épaisseur. La densité de courant de court-circuit Jsc est une figure de mérite de l'efficacité d'absorption du flux solaire et de la collection des charges. Les meilleures performances expérimentales de la littérature (Exp.) restent très proches de ce qui est attendu avec un modèle d'absorption simple ou double passage, alors que les résultats numériques (Num.) prédisent qu'il est possible d'atteindre et même de dépasser le modèle de diffusion lambertienne. Les principales sources de pertes optiques sont représentées en-dessous. 
diffusion sont ajoutés, une partie des photons se retrouve hors du cône de lumière, en réflexion totale interne sur la face avant, augmentant ainsi le chemin optique. Ce piégeage optique améliore l'absorption dans le semiconducteur, mais elle augmente également l'absorption parasite dans les couches de contact. Ainsi, augmenter le nombre d'allers-retours de la lumière n'est efficace que si l'absorption parasite est suffisamment faible. Enfin, les pertes électroniques induites par les recombinaisons non radiatives dans le semiconducteur ou aux interfaces diminuent le rendement quantique externe, et donc le courant Jsc.

Les cellules solaires ultrafines doivent donc combiner un piégeage optique efficace avec de faibles pertes

\section{Aujourd'hui, la diminution de l'épaisseur des cellules solaires peut devenir l'un des éléments clés pour développer des cel- lules solaires à la fois moins chères et plus efficaces.}

optiques et électroniques. Jusqu'à présent, très peu de réalisations sont parvenues à associer l'ensemble de ces contraintes, et on observe les mêmes limitations dans les différentes filières photovoltaïques. Les meilleures performances des cellules solaires conventionnelles et ultrafines sont récapitulées dans le tableau I. Jusqu'à récemment, il fallait plus de $40 \mu \mathrm{m}$ de silicium ou plus d'1 $1 \mu \mathrm{m}$ de semiconducteur en film mince (GaAs, CIGS ou CdTe) pour atteindre une efficacité de $20 \%$.

\section{VERS DES CELLULES SOLAIRES ULTRAFINES ET EFFICACES : $20 \%$ AVEC SEULEMENT 200 NM DE GaAs}

Nous avons démontré récemment une efficacité de conversion solaire de près de $20 \%$ avec seulement $200 \mathrm{~nm}$ de GaAs, grâce à un miroir nanostructuré en face arrière [4]. La structure complète est présentée en figure 3. La recette utilisée est la suivante : les couches semiconductrices sont planaires pour éviter laugmentation des recombinaisons

\section{PIÉGEAGE DE LA LUMIĖRE DANS LES CELLULES SOLAIRES}

On introduit ici trois modèles de référence pour décrire l'absorption et le piégeage optique dans une cellule solaire, schématisée ici simplement sous la forme d'une couche de semiconducteur d'épaisseur $d$, et de coefficient d'absorption $\alpha=4 \pi \kappa / \lambda$ ( $n+i k$ est l'indice de réfraction complexe). $\alpha$ diminue avec l'énergie des photons incidents quand on se rapproche de la bande interdite (généralement dans le proche infrarouge). Pour compenser cette diminution de l'absorption, on peut augmenter le chemin optique dans le dispositif d'un facteur $F$ et écrire l'absorption sous la forme: $A(\lambda)=1-e^{-F a d}$. Avec un revêtement anti-reflet parfait et sans réflexion en face arrière (a), on a un simple passage de la lumière, $F=1$. L'ajout d'un miroir arrière (b) permet un double passage, $F=2$. Comment faire mieux?

La texturation de la face avant de la cellule solaire (c) est le moyen le plus courant d'augmenter le chemin optique par diffusion de la lumière, par exemple par

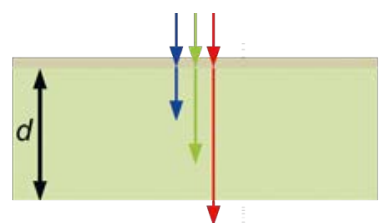

(a) absorption simple passage

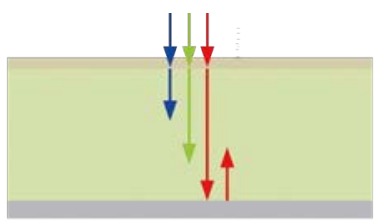

(b) double passage

gravure chimique de la surface (texturation aléatoire). La lumière est déviée puis réfléchie et partiellement piégée par réflexion totale interne. Dans le cas d'un semiconducteur faiblement absorbant comme le silicium, il a été montré que le chemin optique maximal est attendu pour une diffusion lambertienne : $F=4 n^{2}$. Ainsi, le chemin optique peut en théorie être augmenté d'un facteur 50 , soit 25 allers-retours! Dans la pratique, les performances sont encore très éloignées de cette limite théorique (Fig. 1).

Récemment, l'introduction d'une structuration périodique sub-longueur d'onde parfaitement contrôlée (d) a également été explorée dans le but d'améliorer l'absorption grâce à des résonances. La tâche est ardue [3], car de multiples résonances sont nécessaires pour couvrir l'ensemble du spectre solaire. L'optimisation numérique et la fabrication sont également plus compliquées, mais les résultats récents sont prometteurs (Fig. 3).

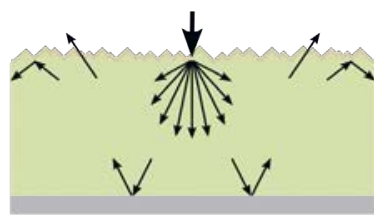

(c) diffusion lambertienne

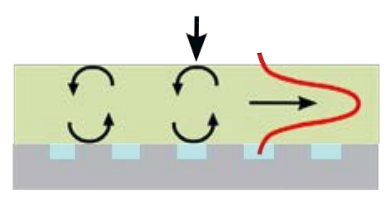

(d) absorption multi-résonnante

Figure 2. Les différentes stratégies de piégeage optique dans une cellule solaire. 
de surface induite par une texturation, et une structuration périodique est utilisée pour coupler la lumière incidente à des modes guidés dans les couches semiconductrices et créer ainsi de multiples résonances aux plus grandes longueurs d'onde, là où l'absorption simple passage est la plus faible. Le nombre de résonances augmente avec la période $p$, mais les pertes par diffraction aux courtes longueurs d'onde aussi (pour $\lambda<p$ ). Le réseau est donc positionné en face arrière, de telle sorte que la diffraction dans l'espace libre n'est possible qu'après un double passage dans l'absorbeur. Une période de $p=700 \mathrm{~nm}$ apparaît comme un bon compromis pour générer de nombreuses résonances dans la région 700-900 nm. La géométrie exacte est optimisée numériquement en résolvant les équations de Maxwell avec la méthode rigorous coupled-wave analysis (RCWA) [5].

Le miroir nanostructuré est formé par nanoimpression d'une couche de $\mathrm{TiO}_{2}$ synthétisée par procédé solgel et recouverte d'argent (Ag), et la face arrière est complétée par des contacts ohmiques localisés (Ni/Ge/ $\mathrm{Au}$ ) qui assurent une extraction des charges avec des pertes résistives minimales, et qui couvrent seulement
$1 \%$ de la surface. Après fabrication de la face arrière, les couches sont transférées, inversées et collées sur un substrat de verre. Le substrat de croissance en GaAs est gravé chimiquement, mais la technique du lift-off permet de le préserver et de le réutiliser pour de nouvelles croissances

Tableau I.

Liste des meilleures performances pour des cellules solaires conventionnelles, fines et ultra-fines. de couches. La fabrication est terminée de manière conventionnelle sur la face avant de la cellule (contacts, mesa et couches anti-reflet). Sur la figure 3, la comparaison de la réponse spectrale obtenue avec un miroir plan (courbe verte) et nanostructuré (rouge) met en évidence le gain d'absorption très significatif obtenu aux grandes longueurs d'onde grâce aux résonances. La précision de la fabrication a permis un excellent accord entre calcul et expérience, et de très bonnes performances électriques.

\begin{tabular}{|c|c|c|c|c|}
\hline TYPE & MATÉRIAU & RÉFÉRENCE & EPAISSEURD & EFFICACITÉ (\%) \\
\hline \multirow{4}{*}{$\begin{array}{c}\text { Records } \\
\text { (cellules épaisses) }\end{array}$} & c-Si & Kaneka 2017 & $160 \mu \mathrm{m}$ & 26.7 \\
\hline & GaAs & $\begin{array}{l}\text { Kayes et al. } \\
2012 \text { / } 2019\end{array}$ & $1-2 \mu \mathrm{m}$ & $28.8 / 29.1$ \\
\hline & CIGS & Wu et al. 2018 & $2-3 \mu \mathrm{m}$ & 22.9 \\
\hline & $\mathrm{CdTe}$ & First Solar 2016 & $3-4 \mu \mathrm{m}$ & 22.1 \\
\hline Fine & c-Si & Kapur et al. 2013 & $40 \mu \mathrm{m}$ & 20.1 \\
\hline \multirow{6}{*}{ Ultrafines } & c-Si & Branham 2015 & $10 \mu \mathrm{m}$ & 15.4 \\
\hline & $\mathrm{c}-\mathrm{Si}$ & Depauw 2017 & $1 \mu \mathrm{m}$ & 8.64 \\
\hline & CIGS & Mansfield 2018 & $490 \mathrm{~nm}$ & 15.15 \\
\hline & \multirow{3}{*}{ GaAs } & Yang et al. 2014 & $300 \mathrm{~nm}$ & 19.1 \\
\hline & & Lee et al. 2015 & $200 \mathrm{~nm}$ & 16.2 \\
\hline & & Chen et al. 2019 & $200 \mathrm{~nm}$ & 19.9 \\
\hline
\end{tabular}

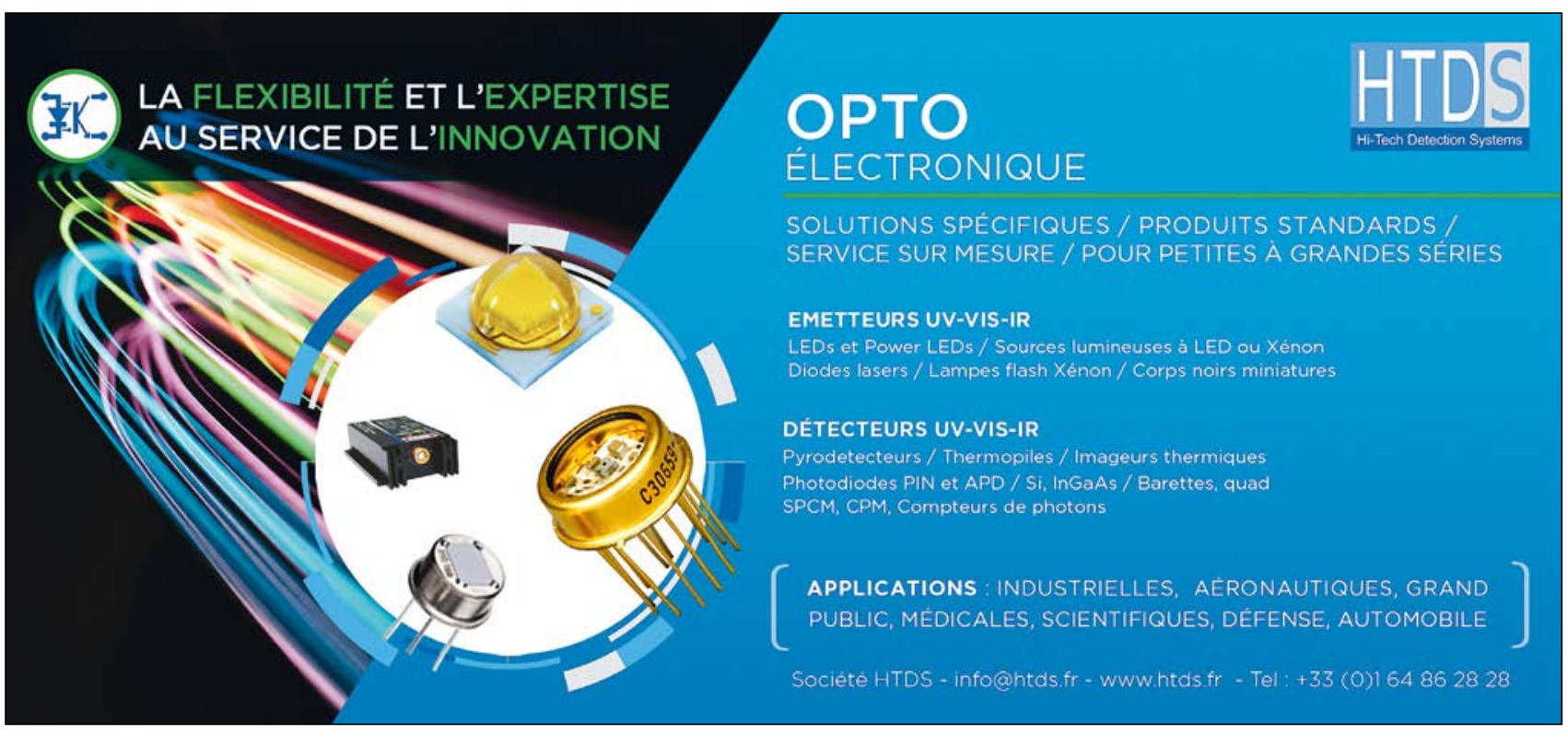



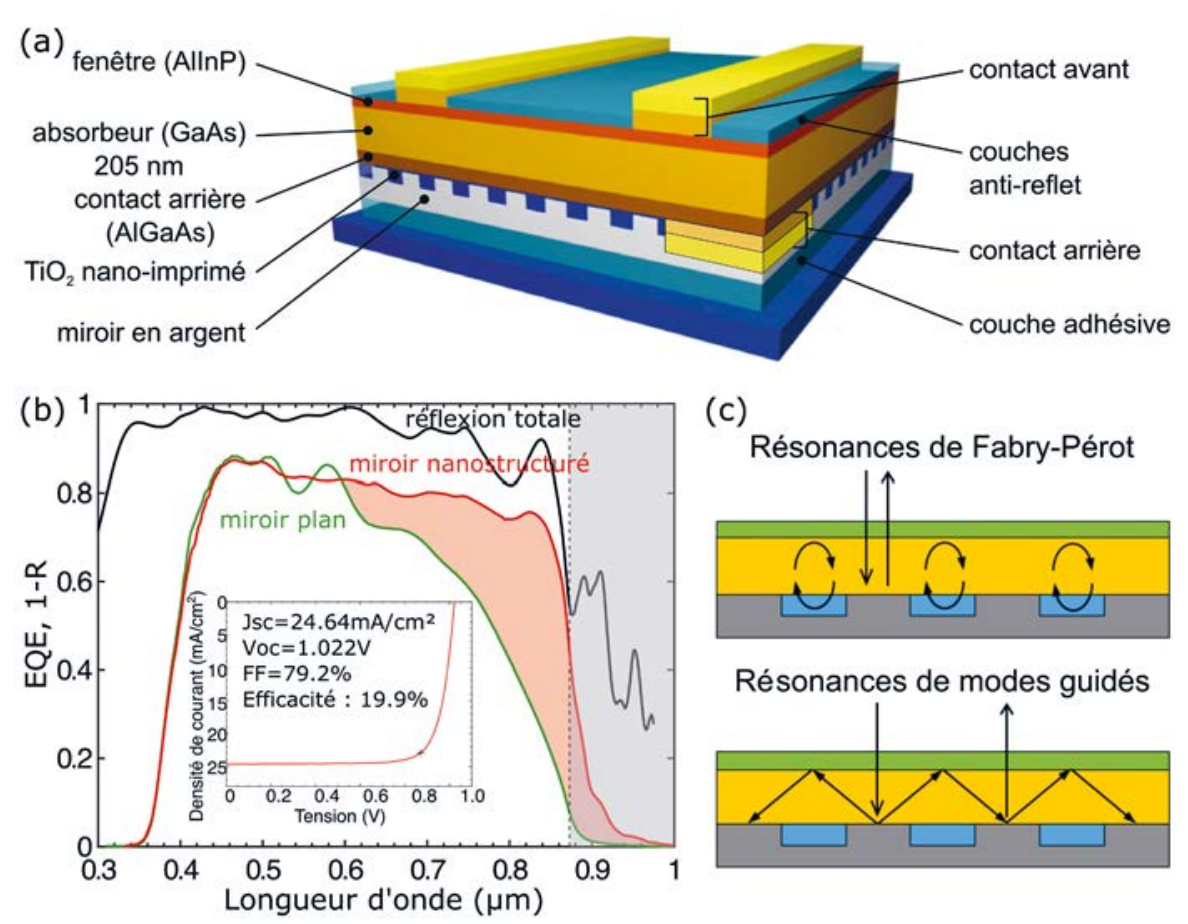

(c)

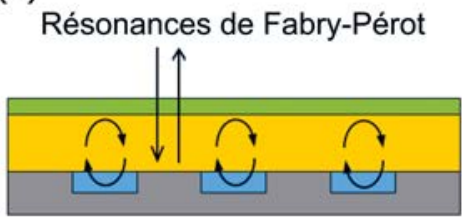

Résonances de modes guidés

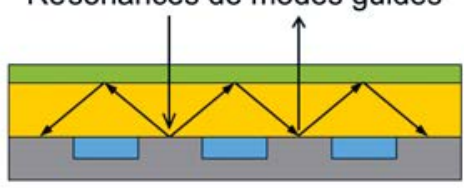

Figure 3.

Cellule solaire ultrafine formée de 200 nm de GaAs. Un miroir nanostructuré en face arrière permet de créer des résonances multiples dans la bande spectrale 700-900 nm. Le gain d'absorption par rapport à un miroir arrière plan est représenté par la zone rouge sur les spectres de rendement quantique externe (EQE) (b), et permet d'atteindre une efficacité de conversion de $19,9 \%$ sous illumination solaire. II est possible d'identifier les résonances mises en jeu à des résonances de type Fabry-Pérot et de modes guidés (c)

remplacer la fabrication de cellules à partir de wafers fabriqués par sciage, source de pertes. Dans le cas des couches minces (CdTe, CIGS), la diminution de l'épaisseur a un impact immédiat sur les temps de dépôt, et donc sur la productivité et les coûts d'investissement des usines. Elle permet également une économie d'éléments rares comme le tellure ou l'indium. Les écueils technologiques restent importants, et nécessitent le développement de procédés qui doivent être impérativement très bas coût, mais l'activité de recherche sur le CIGS ultrafin est particulièrement active en France et en Europe, notamment au sein du consortium ARCIGS-M qui regroupe laboratoires académiques et industriels. travaux théoriques, numériques et expérimentaux depuis une dizaine d'années. En conciliant nanophotonique et photovoltaïque, les résultats récents ont montré que les cellules solaires ultrafines peuvent atteindre des efficacités supérieures à $20 \%$. Ils ouvrent des perspectives d'application dans différents domaines. La faible épaisseur offre une plus grande tolérance aux défauts de l'absorbeur. Dans le domaine spatial, une plus grande durée de vie

est donc attendue pour ces cellules solaires III-V soumises au bombardement de particules très énergétiques. Cette tolérance permet également d'utiliser des matériaux de moindre qualité, synthétisés avec des procédés bas coût. Par exemple, l'épitaxie de silicium en phase vapeur pourrait

\section{RÉFÉRENCES}

[1] S. Kurtz et al., Nat. Photon. 11, 3 (2017)

[2] D. Lincot, Photoniques 93, 37 (2018)

[3] S. Collin, A. Cattoni, Photoniques 90, 25 (2018)

[4] H.-L. Chen et al., Nature Energy 4, 761 (2019)

[5] M. Besbes, J.-P. Hugonin, C. Sauvan, Photoniques 100, 34 (2020) 